\title{
Docencia e Investigación en Odontología Legal en Chile: Situación y Desafíos Actuales
}

\author{
Teaching and Research in Legal Dentistry in Chile: Current Situation and Challenges
}

\author{
Denisse Lagos Tissie ${ }^{1,2}$; Lidia Bravo R. '; José Luis Ramos Moreno ${ }^{3,4}$ \& Luis Ciocca Gómez
}

LAGOS, T. D.; BRAVO, R. L.; RAMOS, M. J. L. \& CIOCCA, G. L. Docencia e investigación en odontología legal en Chile: Situación y desafíos actuales. Int. J. Odontostomat., 12(3):274-279, 2018.

RESUMEN: El objetivo de este estudio es describir las características de las instituciones y el equipo de investigación y docencia que imparten Odontología Legal (OL) a nivel pregrado en Chile. Estudio cualitativo realizado durante el 2017. Desde las páginas web institucionales de las universidades que imparten la carrera de odontología en Chile, se obtuvo información sobre el cuerpo docente del ramo de OL a nivel pregrado. Cuando la información no estuvo disponible, se solicitó vía correo electrónico. Se realizó una búsqueda de publicaciones chilenas sobre OL y Forense en las bases de datos Medline, SciELO, Lilacs y Cochrane, y se identificó aquellas realizadas por docentes ejerciendo en Chile. La asignatura de OL se imparte a nivel pregrado en 17 de las 21 universidades que imparten la carrera de Odontología, siendo un curso obligatorio en 16 de ellas, y electivo en una de ellas. De los 29 docentes que imparten el ramo a nivel pregrado en Chile, sólo tres tiene la especialidad de OL. Se encontraron sólo 28 publicaciones científicas en el área en los últimos diez años, cuya autoría corresponde a cinco de los docentes del ramo a nivel pregrado. En Chile, la docencia de $\mathrm{OL}$, a nivel pregrado, es impartida mayoritariamente por cirujano-dentistas no especializados en el área, en concordancia con que la especialidad comenzó a impartirse en el año 2015. Es imperioso aumentar la investigación en OL entre los docentes, de manera de desarrollar el área a nivel universitario, aumentando el conocimiento en la disciplina y consolidándola como ciencia.

PALABRAS CLAVE: enseñanza en odontología, odontología forense, investigación en odontología, competencia profesional.

\section{INTRODUCCIÓN}

La Odontología Legal (OL) vincula al cirujanodentista con la ley, quien aporta su conocimiento en la resolución de casos judiciales en las áreas de identificación humana, lesionología, criminalística, marco regulatorio del ejercicio odontológico y responsabilidad profesional, además de participar en la creación y aplicación de la reglamentación en relación a políticas de salud pública, como en enfermedades y accidentes laborales, y de la ética y bioética en la práctica profesional (Committee on Identifying the Needs of the Forensic Science Community, 2009; Ciocca, 2010; Ortiz et al., 2015).
El comienzo de la disciplina en Chile data de 1909, año del incendio de la Legación Alemana, donde la odontología fue fundamental en la labor identificatoria propia del ámbito forense (Palacios, 2015). Sin embargo, su enseñanza en las escuelas de pregrado comienza recién en 1992, en la Universidad de Chile (Ciocca). La formación de postítulo en el área contempla un Diplomado en Odontología Forense dictado anualmente por la Universidad de la Frontera desde el año 2015 y el primer Programa de Formación de Especialistas en Odontología Legal y Forense, que comenzó a impartirse el año 2015 en la Universidad de Chile.

\footnotetext{
${ }^{1}$ Especialista en Odontología Legal y Forense, Medicina Legal Odontológica, Facultad de Odontología, Universidad de Chile. Santiago, Chile.

${ }^{2}$ Odontología Legal, Facultad de Odontología, Universidad Pedro de Valdivia, Santiago, Chile.

${ }^{3}$ Especialista en Imagenología, Especialista en Odontología Legal.

${ }^{4}$ Dirección General de Aeronáutica Civil (DGAC), Medicina Legal Odontológica, Facultad de Odontología, Universidad de Chile. Santiago, Chile.

${ }^{5}$ Especialista en Ortodoncia y Ortopedia Dentomaxilofacial, Especialista en Odontología Legal. Medicina Legal Odontológica, Facultad de Odontología, Universidad de Chile. Santiago, Chile.
} 
Actualmente, la carrera de Odontología en Chile se imparte en 21 universidades y tiene una duración de doce semestres en 19 de estas instituciones, y de 11 semestres en las dos restantes. Dada la variedad de instituciones que ofrecen la carrera, la homogenización de los contenidos impartidos en cada asignatura es difícil de lograr. En el caso particular de la OL, el año 2015 la secretaría del Instituto Dr. Carlos Ybar del Servicio Médico Legal de Chile, convocó a docentes del área pertenecientes a universidades integrantes de la Asociación Chilena de Enseñanza de la Odontología (ACHEO), con el fin de conformar un grupo de trabajo para revisar y proponer los contenidos mínimos de la asignatura de OL que se debieran impartir en las distintas casas de estudios. La mesa de trabajo, que reunió a 12 universidades chilenas, informó que los temas más tratados en las cátedras respectivas son: lesionología (92\% de las universidades), responsabilidad profesional (83\%), identificación $(75 \%)$ y consentimiento informado e historia clínica (58 \% cada uno) (Faundes et al., 2017).

Junto con los contenidos que debe tener la asignatura, el otro factor determinante en la calidad de un programa, es la idoneidad de quienes los imparten, pues son los responsables de que éste cubra todas las áreas del conocimiento necesarias para dotar al estudiante con las competencias necesarias para desempeñarse en el futuro en su vida laboral.

El propósito de este estudio es describir las características del equipo de investigación y docencia que imparten Odontología Legal en Chile.

\section{MATERIAL Y MÉTODO}

\section{Diseño: Se realizó un estudio cualitativo de corte transversal.}

Fuentes de información e instrumentos de recolección: Del universo de las 60 universidades nacionales reconocidas por el Estado al año 2017, se consideró a las 21 universidades que imparten la carrera de odontología (Ministerio de Educación, 2017). Desde sus páginas web institucionales se extrajo la información sobre el cuerpo docente del ramo de OL en aquellas universidades que impartían efectivamente dicho ramo.

Cuando la información no estuvo disponible, se solicitó vía correo electrónico a las autoridades res- pectivas, señalando las características del estudio y asegurando el manejo confidencial de la información considerada sensible según la Ley 19.628 (Diario Oficial de la República de Chile, 1999). La especialidad de los docentes fue obtenida de la base del Registro de Prestadores Individuales en Salud (Superintendencia de Salud, 2018).

Finalmente, se realizó una revisión bibliográfica de las publicaciones chilenas en el área de la Odontología Legal y Forense, utilizando las bases de datos bibliográficos Lilacs, Cochrane, SciELO y Medline. Se consideraron como publicaciones chilenas, aquellas en que la filiación de uno o más autores correspondiera a cualquiera institución chilena, universitaria o auxiliar de la justicia. Dentro del total de las publicaciones encontradas, se identificó a aquellos autores que realizan docencia de la materia en Chile.

Los datos recogidos fueron tabulados en planilla Microsoft Excel 2007® y se procedió a un análisis descriptivo de los resultados, para lo cual se recurrió a medidas de resumen y tablas. Para la búsqueda se utilizaron los términos Mesh "forensic odontology", "forensic dentistry", "odontología legal" y "odontología forense"

\section{RESULTADOS}

La carrera de Odontología se imparte en 21 universidades chilenas. El ramo de "Odontología Legal" es también llamado "Odontología Legal y Forense", "Odontología y Medicina Legal" o "Medicina Legal Odontológica" y se imparte en un total de 17 universidades. Se recabó la información completa de 15 de estas universidades, mientras que de una universidad se obtuvo sólo información parcial y de otra no se obtuvo información por los medios definidos en la metodología. En 16 de las 17 universidades el ramo es parte de la malla curricular de manera obligatoria, y en una, el ramo es electivo. En todas las universidades que imparten la asignatura, ésta es de carácter semestral, siendo impartida mayoritariamente $(58,8 \%)$ en el quinto año de la carrera (Tabla I).

Respecto al cuerpo docente que imparte el ramo en los distintos planteles, y sobre un universo de 29 profesores, encontramos que son en su mayoría cirujano dentistas, siendo sólo dos de ellos de una profesión distinta: un abogado y un médico cirujano especialista en Medicina Legal. Entre los 27 odontólogos, 
tenemos que 14 (52\%) no registran ninguna especialidad en el Registro de Prestadores Individuales en Salud, 3 (11\%) tienen la especialidad en Odontología Legal y Forense, 2 son especialistas en imagenología, 2 especialistas en Periodoncia, 2 especialistas en $\mathrm{Pa}-$ tología Oral y 2 en Salud Pública $(7,4 \%$ cada especialidad). Las especialidades de Rehabilitación Oral, Implantología y Cirugía Máxilofacial están representadas con un docente cada una $(3,7 \%$ cada especialidad). Además, 6 de ellos se desempeñan en instituciones auxiliares de la justicia: 5 en el Servicio Médico Legal y uno en Carabineros de Chile.

La búsqueda en las bases de datos de revistas científicas arrojó un total de 2477 estudios relacionados con Odontología Legal y Forense en los últimos 10 años a nivel mundial. En ese mismo periodo, hubo 47 publicaciones en la materia realizadas por investigadores afiliados a alguna Institución chilena. De estas 47 publicaciones, $28(59,6 \%)$ son de autoría de alguno de sólo 5 de los docentes que imparten el ramo en Chile (Fig. 1). Aún más; de los 28 artículos, 21 son de autoría de uno de estos docentes. Los cinco docentes autores se desempeñan como académicos e investigadores en sus respectivos planteles universitarios y no pertenecen a ninguna de las instituciones auxiliares de la justicia.

Respecto a los temas tratados en dichas publicaciones, 16 están relacionados con identificación (10 sobre métodos odontológicos de identificación, 5 sobre perfil biológico y uno sobre patología forense), 6 con criminalística (3 sobre cambios experimentados por las piezas dentales o materiales de restauración en el período postmortem, y 3 sobre veterinaria forense), 4 tratan de temas relacionados con el profesionalismo, uno sobre ética en la atención clínica, y uno sobre seguridad en la atención del paciente.

Cabe destacar también que, de los 29 docentes del ramo, 3 han publicado libros y/o textos de estudios del tema.

Tabla I. Universidades chilenas que imparten la carrera de Odontología y las características del ramo de Odontología Legal.

\begin{tabular}{|c|c|c|}
\hline Universi dad & $\begin{array}{c}\text { Asignatura OL } \\
\text { (semestre) }\end{array}$ & $\begin{array}{c}\mathrm{N}^{\circ} \\
\text { docentes OL }\end{array}$ \\
\hline \multicolumn{3}{|l|}{ Universidades del Consejo de Rectores } \\
\hline Pontifica Universidad Católica de Chile & $\mathrm{XI}$, obligatoria & 3 \\
\hline Universidad Arturo Prat & IX, obligatoria & 1 \\
\hline Universidad Austral de Chile & VII, obligatoria & 1 \\
\hline Universidad de Antofagasta & $\mathrm{XI}$, obligatoria & 2 \\
\hline Universidad de Chile & $\mathrm{X}$, obligatoria & 4 \\
\hline Universidad de Concepción & No se imparte & - \\
\hline Universidad de La Frontera & IX, electiva & 1 \\
\hline Universidad de La Serena & IX, obligatoria & Sin información \\
\hline Universidad de Talca & No se imparte & - \\
\hline Universidad de Valparaíso & $\mathrm{XI}^{*}$ & 1 \\
\hline \multicolumn{3}{|l|}{ Universidades Privadas } \\
\hline Universidad Andrés Bello & X, obligatoria & 3 \\
\hline Universidad Autónoma de Chile & No se imparte & - \\
\hline Universidad Bolivariana & No se imparte & - \\
\hline Universidad de Los Andes & VII, obligatoria & 3 \\
\hline Universidad de Viña del Mar & $\mathrm{X}$, obligatoria & 1 \\
\hline Universidad del Desarrollo & IX, obligatoria & 2 \\
\hline Universidad Diego Portales & $\mathrm{X}$, obligatoria & 2 \\
\hline Universidad Finis Terrae & VIII, obligatoria & 1 \\
\hline Universidad Mayor & $\mathrm{X}$, obligatoria & 2 \\
\hline Universidad Pedro de Valdivia & IX, obligatoria & 4 \\
\hline Universidad San Sebastián & XII, obligatoria & $2^{* *}$ \\
\hline
\end{tabular}

\section{DISCUSIÓN}

El presente, es el primer estudio que se realiza en Chile sobre docencia en Odontología Legal y con- sideramos que varios hallazgos resultan interesantes de discutir: 


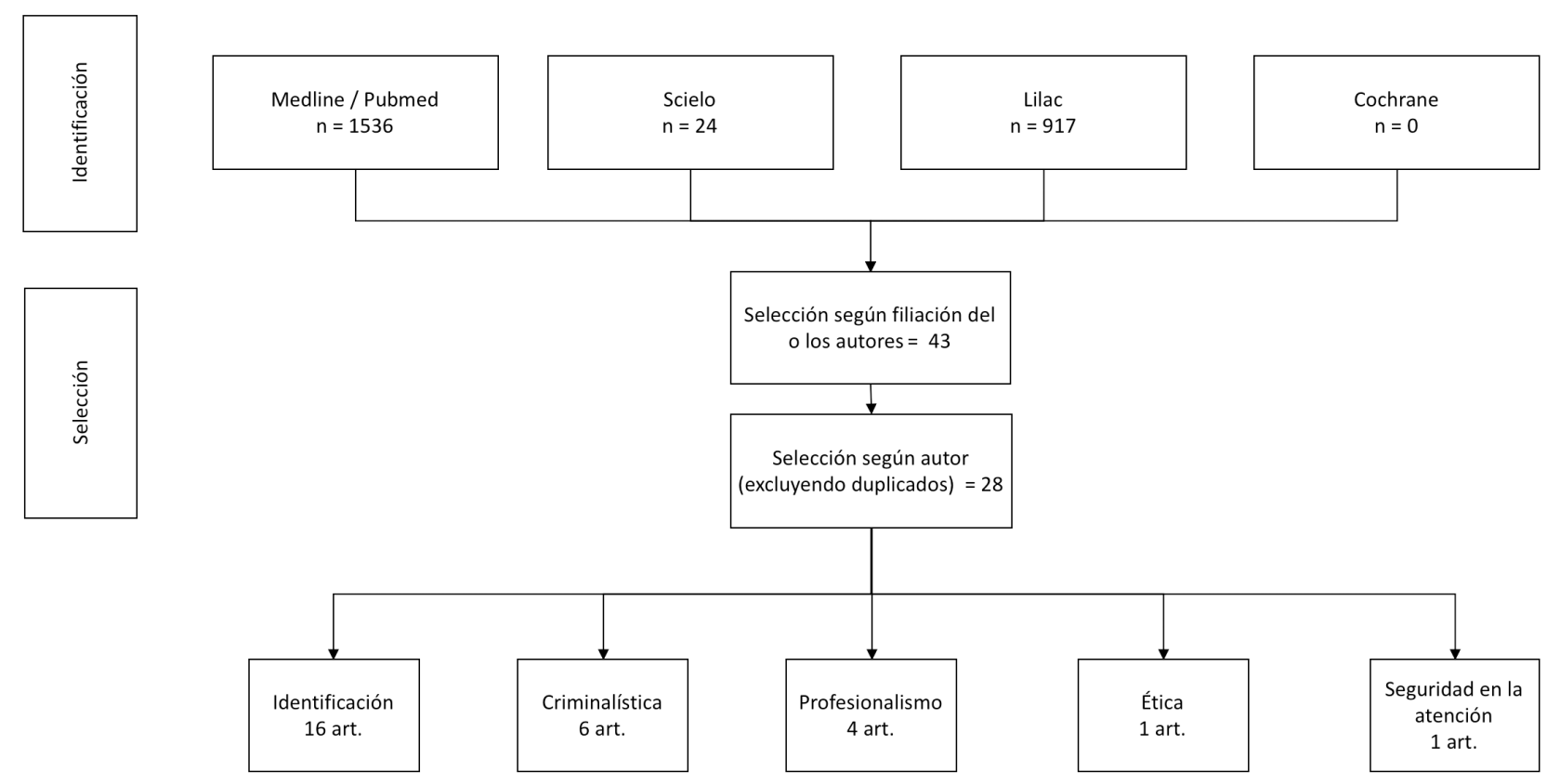

Fig. 1. Diagrama de la revisión sistemática realizada en la búsqueda de documentos sobre Odontología Legal escritos por docentes deOdontología Legal. Se utilizaron los siguientes descriptores o términos Mesh de búsqueda: en Medline/PubMed, "forensic odontology OR forensic dentistry"; en SciELO, "odontología legal", "odontología forense", "forensic dentistry" y "forensic odontology"; en Lilacs, "odontología AND legal" y "odontología AND forense"; y en la base de datos Cochrane, "Forensic dentistry".

Impartición de Odontología Legal dentro de la malla curricular. En casi un cuarto de las universidades en que se imparte la carrera de Odontología $(23,8 \%)$ el ramo de OL no se imparte o no es obligatorio. Considerando lo relevante que son las temáticas que se abordan en el ramo para el ejercicio habitual de la profesión, resulta preocupante esta cifra. Hoy en día, junto a la ya consabida judicialización de la práctica médica y el aumento de las demandas por malpraxis, la necesidad de contar con especialistas en el área de salud laboral, sumado a la importancia que han cobrado los peritajes científicos en el nuevo sistema penal chileno, pone de manifiesto la importancia de que los conocimientos entregados a los estudiantes de pregrado cubran el amplio abanico de temas que componen la OL, además de los temas preferentemente tratados hoy en las cátedras de pregrado.

Otro punto importante a comentar es la oportunidad en que se imparte el ramo. En uno de los 17 planteles que imparten la asignatura, ésta es dictada en el último semestre de la carrera, posterior al internado docente asistencial o práctica profesional, con lo que estos alumnos realizan su primer acercamiento al mundo laboral profesional, sin haber recibido todos los conocimientos que necesita para enfrentar el trabajo con pacientes.
Formación de los docentes que imparten Odontología Legal. La información entregada a los estudiantes en su formación de pregrado debe estar acorde a los avances científicos de la disciplina a nivel global y en concordancia con las necesidades de la sociedad. En el caso de la OL en particular, la realidad local no dista demasiado de la del resto del mundo: un aumento de la judicialización de los inevitables conflictos suscitados en la práctica clínica que nos obliga a preparar a nuestros estudiantes tanto en la prevención de problemas en la relación médico paciente, como a saber qué hacer frente a los mismos. De ahí la importancia de contar con docentes idóneos, que conozcan el sistema judicial del país y orienten a los futuros dentistas en temas relativos a la responsabilidad médica. En este estudio constatamos que, de los 29 docentes que imparten el ramo en las universidades chilenas, sólo 3 tienen la especialidad en Odontología Legal y Forense, uno cuenta con la especialidad en Medicina Legal, y otros 6 se desempeñan en instituciones auxiliares de la justicia. Esto guarda relación con el hecho de que la especialidad comenzó a impartirse en Chile sólo el año 2015.

Investigación en Odontología Legal. Llama la atención también que sólo 5 de los docentes hayan realizado publicaciones en los últimos 10 años. La investigación y extensión han sido tradicionalmente el pilar del 
desarrollo de las instituciones de educación superior modernas, por sobre la docencia. En una breve síntesis histórica, encontramos que a finales del siglo XIX, según el modelo de Humboldt, se introduce la ciencia y la investigación en el quehacer universitario, en un afán de la monarquía de eliminar la tutela de la iglesia en la producción de conocimientos. Este modelo alemán, fue imitado por las universidades norteamericanas desde 1876 y a principios del s.XX por universidades latinoamericanas. En 1949, en el Primer Congreso de Universidades Latinoamericanas realizado en Guatemala, se aprobaron una serie de reformas referidas a la acción social de la universidad y la extensión cultural universitaria, señalándose que la universidad debe estar al servicio de la comunidad, contribuyendo a la resolución los problemas de la nación, a partir de la función de extensión (Sira, 2011). La educación impartida hoy en algunas universidades ha priorizado la docencia por sobre la investigación y extensión. La investigación es la base de la generación de nuevo conocimiento, el cual es luego transmitido a los alumnos y a la comunidad. Las ciencias forenses en general, y la OL en particular, no debiesen estar ajenas a esto y debiesen procurar enfocarse en un aumento de la investigación de los temas que le son propios, de modo de consolidarse como ciencia. Creemos que esto también debiese interesar a quienes se desempañan en organismos auxiliares de la justicia, aportando desde su tribuna a la generación de conocimiento y a la justicia.

Relevancia de investigar en las distintas áreas de la Odontología Legal. Constatamos la carencia de investigación en Chile en áreas tan relevantes como accidentes de trabajo, enfermedades laborales, enfermedades de notificación obligatoria, garantías de acceso a salud oral, problemas musculoesqueléticos sufridos por el odontólogo o la pesquisa de lesiones por violencia intrafamiliar en el área maxilofacial, ya que no son ajenos a nuestro ejercicio y son temas propios de esta área de la OL. Una mayor investigación en este sentido generaría nuevo conocimiento que podría ser entregado a nuestros alumnos en su formación de pregrado.

Creemos también que, si bien el aumento de las demandas por malpraxis médica es una realidad que nos obliga a replantear los contenidos impartidos en la carrera de pregrado, no podemos desconocer ni dejar de lado los conocimientos sobre ciencias forenses, particularmente los que se refieren a identificación en distintos contextos. Los aportes de la odontología en esta área son indiscutidos, dadas las características de las piezas dentales y demás estructu- ras maxilofaciales y de los materiales de restauración usados en nuestra práctica clínica habitual. Chile es un país que se ve expuesto frecuentemente a desastres naturales, por lo que el cirujano dentista formado en el país debería poseer los conocimientos básicos para actuar en casos de desastres masivos. Al respecto, Hermsen \& Johnson (2012) propusieron que un adecuado programa de pregrado orientado a las ciencias forenses en odontología, debiese contener los siguientes tópicos: una visión general de la odontología forense, métodos más usados en la odontología forense actual, rol del cirujano dentista en la respuesta ante desastres masivos y actualización en temas forenses posterior a su egreso de la carrera. Adaptar dichos contenidos a la realidad local, podría resultar un aporte a la formación de pregrado.

Hay que considerar, además, el papel relevante que juega a diario el odontólogo en la acreditación o constatación de lesiones. En más de alguna ocasión, el cirujano dentista se verá enfrentado a pacientes con lesiones en el territorio maxilofacial producto de accidentes de tránsito, riñas, violencia intrafamiliar, entre otras situaciones que requerirán de sus conocimientos expertos para ser constatadas. Esta primera evaluación es fundamental para que el perito en lesionología forense se pronuncie posteriormente respecto del pronóstico médico legal de las lesiones, señalando el tiempo que demorarán en sanar o la incapacidad laboral que generan, además de vincularlas causalmente con los hechos delictivos investigados. Al respecto, Contreras \& Hermosilla (2014), concluyeron en su estudio, que existe un sub-registro de lesiones odontológicas y maxilofaciales en esta primera evaluación del paciente, lo cual determina una falta de elementos para llegar a una correcta administración de justicia, en la aplicación de penas y/o compensación de daños al afectado. Por lo anteriormente expuesto, es también imperativo instruir a los alumnos de pregrado en los aspectos no sólo clínico de las lesiones, sino también en los aspectos legales y las implicancias de esta primera evaluación.

Limitaciones del estudio. Si bien el año 2015 se realizó una mesa de trabajo con el propósito de proponer un programa de contenidos mínimos a impartir en el ramo de OL en las carreras de pregrado en Odontología en Chile, tal como se citó en la introducción, los resultados obtenidos no fueron del todo los esperados, pues sólo se logró extraer información de tipo cualitativo de los respectivos programas de asignatura. Contar con información sobre la cantidad exacta de horas pedagógicas dedicadas a cada subtema impartido en la 
cátedra de OL en cada una de las universidades nacionales, permitiría realizar un análisis cuantitativo y comparativo entre los mismos, pudiendo tener un panorama más amplio de la realidad local y proponer un programa estandarizado a impartir a nivel nacional.

En el presente estudio, la información limitada entregada por uno de los planteles, y la no respuesta de otro, hacen que el presente estudio pueda sacar sólo conclusiones parciales respecto a los temas investigados.

\section{CONCLUSIÓN}

En docencia universitaria es trascendental contar con profesores idóneos, que estén al día en los últimos avances y aplicaciones de sus respectivas ciencias. En Chile, la docencia de la OL, a nivel pregrado, es impartida mayoritariamente por cirujano dentistas no especializados en el área, haciéndose imperiosa la necesidad de avanzar en ese sentido. Es preciso aumentar la investigación en OL entre los docentes, desarrollando el conocimiento en la disciplina y consolidándola como ciencia. Un completo programa de pregrado en OL, debería incluir la mayor cantidad de tópicos que le son propios al área y estar orientado a las necesidades de la comunidad en que se desempeñará el futuro odontólogo.

LAGOS, T. D.; BRAVO, R.L.; RAMOS, M. J. L. \& CIOCCA, G. L. Teaching and research in legal dentistry in Chile: current situation and challenges. Int. J. Odontostomat., 12(3):275-279, 2018.

ABSTRACT: The aim of this study was to describe the characteristics of the universities and the research and education teams imparting "Forensic Odontology" at undergraduate level in Chile. A qualitative cross-sectional study was conducted during 2017. Information was sought from the institutional websites of universities that impart Dentistry degrees. When the information was not available, it was requested via email to the respective authorities. A search was conducted to identify Chilean publications that deal with the topic of forensic dentistry, in the Medline, SciELO, Lilacs and Cochrane databases. Those authored or co-authored by academic(s) teaching in Chile were identified. "Forensic Odontology" is taught at undergraduate level in 17 of the 21 universities that impart the career of Dentistry in Chile, being mandatory in 16 of schools, and an elective in one of them. Of the 29 academics who teach the subject at undergraduate level in Chile, only three have the specialty of OL. Only 28 scientific publications in the area were found in the last ten years, whose authorships correspond to five academics in the field, teaching at undergraduate level. In Chile, "Forensic Odontology" at the undergraduate level, is mainly imparted by dental surgeons not specialized in the subject, in accordance with the fact that the specialty began to be taught in Chile only in 2015. It is imperative to increase the amount of research in forensic odontology conducted by the academics, in order to develop the area at the university level, to improve knowledge in this discipline and consolidate it as a science.

KEY WORDS: dental education, forensic odontology, dental research, professional competence.

\section{REFERENCIAS BIBLIOGRÁFICAS}

Ciocca, L. Odontología Médico Legal. Santiago de Chile, Ediciones Jurídicas de Santiago, 2010.

Committee on Identifying the Needs of the Forensic Science Community. Strengthening Forensic Science in the United States. A Path Forward. Washington D. C., The National Academies Press, 2009.

Contreras, C. \& Hermosilla, B. Acreditación de lesiones y trauma dentoalveolar: un desafío. Investig. Forense, 3(1):66-73, 2014.

Faundes, M.; Carrasco, P. \& Lagos, D. Estado de la Enseñanza de la Odontología Legal en Chile. Santiago de Chile, Congreso Iberoamericano de Ciencias Forenses, 2017.

Hermsen, K. P. \& Johnson, J. D. A model for forensic dental education in the predoctoral dental school curriculum. J. Dent. Educ., 76(5):553-61, 2012.

Ley 19.628. Sobre Protección de la Vida Privada. Santiago de Chile, Diario Oficial de la República de Chile, 1999.

Ministerio de Educación. Listado de Instituciones de Educación Superior reconocidas por el Estado. Santiago de Chile, Ministerio de Educación, 2017. Disponible en: http:// portal.beneficiosestudiantiles.cl/sites/default/files/ iesreconocidas2017.pdf

Ortiz, J; Fonseca, G. M.; Cantín, M.; Huitzil, E. \& Lucena, J. Generic skills for education in forensic dentistry: Critical thinking and social responsibility. Int. J. Odontostomat, 9(2):263-72, 2015.

Palacios, C. Crónica: Guillermo Beckert. En: Palacios, C. \& Leyton, C. (Eds.). Industria del Delito: Historia de las Ciencias Criminológicas en Chile. Santiago de Chile, Museo Nacional de Odontología, Facultad de Odontología, Universidad de Chile, Ocho Libros Editores, 2015. pp.151-6.

Sira, S. Equilibrio entre las funciones de docencia, investigación y extensión en ingeniería. Desarrollo histórico. Ing. Soc. U. C., $6(1): 51-7,2011$

Superintendecia de Salud. Registro Nacional de Prestadores Individuales de Salud. Santiago de Chile, Superintendecia de Salud, Ministerio de Salud, Gobierno de Chile, 2018. Disponible en : http:/ / we bhosting.superdesalud.gob.cl/base s/ prestadoresindividuales.nsf/buscador?openForm

Dirección para correspondencia:

Dra. Denisse Lagos Tissie

Facultad de Odontología

Universidad de Chile

Santiago - CHILE

Recibido : 06-05-2018

Aceptado: 14-06-2018

Email: dlagos@odontologia.uchile.cl 